\title{
Aplicación para el monitoreo colaborativo de la calidad de cuerpos de agua usando bioindicadores
}

\author{
Irving Galindo-Márquez, Ángel López-Sánchez, Luis Hernández-Mercado, \\ Carlos Sosa-Paz, Juan-Pablo Posadas-Durán \\ Instituto Politécnico Nacional, Escuela Superior de Ingeniería Mecánica y Eléctrica, \\ Unidad Zacatenco (ESIME Zacatenco), México \\ jdposadas@esimez.mx, omar.syn10@gmail.com, enriquehm97@gmail.com, \\ csosapaz@gmail.com
}

\begin{abstract}
Resumen. El monitoreo colaborativo móvil (Mobile Crowd Sensing) es una técnica de monitoreo que se basa en la participación de personas que usan algún dispositivo móvil (teléfonos inteligentes, tabletas, etc.) para colectar datos de diversos tipos del entorno en que se encuentran y los transmiten hacia un nodo central para medir, georreferenciar, analizar y estimar procesos de interés. En este trabajo se propone un prototipo de plataforma móvil para la evaluación y monitoreo de la calidad de cuerpos de agua, mediante el uso de teléfonos inteligentes, que permita evaluar el cambio temporal de la calidad de cuerpos de agua por medio de bioindicadores utilizando el índice BMWP. El prototipo, además de utilizar estrategias de monitoreo colaborativo, realiza un análisis de los datos recolectados para determinar el estado del cuerpo de agua e indicar el nivel de participación de los colaboradores.
\end{abstract}

Palabras clave: crowdsensing, dispositivos móviles, internet de las cosas, ciudades inteligentes.

\section{Crowdsensing App for Monitoring Quality of Water Bodies Using Bioindicators}

\begin{abstract}
Mobile Crowd Sensing is a monitoring technique that relies on the participation of people who use a mobile device (smartphones, tablets, etc.) to collect data from various types of the environment and transmit them to a central node to measure, georeference, analyze and estimate processes of interest. This work proposes a prototype of a mobile platform for the evaluation and monitoring of the quality of water bodies, using bioindicators described in the BMWP index. The prototype also performs an analysis of the data collected to determine the status of the body of water and indicates the level of participation of the collaborators.
\end{abstract}

Keywords: crowdsensing, mobile devices, IoT, smart cities. 
Irving Galindo-Márquez, Ángel López-Sánchez, Luis Hernández-Mercado, Carlos Sosa-Paz, et al.

\section{Introducción}

México se encuentra en transición hacia una sociedad de la información, en la que las Tecnologías de la Información y Comunicación (TIC), son utilizadas con frecuencia por la sociedad para realizar la mayor parte de sus actividades. Esta integración permite acceder a información o servicios ofrecidos local o internacionalmente, mediante el uso de diversos dispositivos además de las computadoras. El nivel de integración de las TIC en una sociedad es un indicador del desarrollo de un país, además le permite participar en políticas o programas a nivel global.

En México, empresas y diversos sectores del gobierno como el educativo, medio ambiente, salud y seguridad, por mencionar algunos, requieren de acciones que les permitan iniciar o aumentar su nivel de integración de las TIC, preferentemente hasta un nivel como el que se tiene en países desarrollados. Las TIC son un conjunto de herramientas tecnológicas utilizadas para procesar y administrar información de manera eficiente; abarcan a las telecomunicaciones y diversas áreas de la computación como son tecnologías de la informática, tecnologías de software, programación avanzada, inteligencia artificial, interfaces hombre-máquina, ciberseguridad, bases de datos, entre otras.

En el presente documento, se describe una propuesta de infraestructura que tiene como objetivo desarrollar un sistema informático, que permita recopilar información sobre la calidad de cuerpos de agua en México, mediante el uso de teléfonos inteligentes con la participación de los pobladores de las comunidades cercanas a los cuerpos de agua. La evaluación del cuerpo de agua se realizará a través de una encuesta que implemente el método BMWP para conocer el índice que calidad de cuerpos de agua. La encuesta se implementará como una aplicación para teléfonos inteligentes. Una vez recopilada la información mediante la aplicación por parte de los participantes se almacenará en un servidor central. El sistema informático permitirá a un experto acceder a la información recopilada para su valoración y posterior integración en una base de datos. Existirá una retroalimentación e información mostrada en páginas Web dinámicas, a los pobladores y autoridades locales y federales, sobre el estado que guarda cada cuerpo de agua georreferenciado mediante páginas Web dinámicas que mostrarán indicadores sobre la calidad de los cuerpos de agua.

El resto del artículo se organiza de la siguiente forma: en la Sección 1 se muestra una breve descripción de las características sobre el monitoreo colaborativo móvil, en la Sección 2 se describe brevemente el índice de calidad del agua mediante el paradigma de BMWP que se basa en la identificación de organismos macroinvertebrados en los cuerpos; en la Sección 3 se describe la propuesta para el desarrollo de la aplicación y en la Sección 4 se describen las conclusiones.

\section{Monitoreo colaborativo móvil}

El Monitoreo Colaborativo Móvil (en inglés, Mobile Crowd Sensing, MCS), hace referencia a un paradigma en el que se utilizan dispositivos móviles para recolectar información sobre un fenómeno de interés y compartir esta información 
con sistemas de cómputo a través del Internet. En el MCS intervienen un conjunto de participantes distribuidos geográficamente con la finalidad de realizar una tarea de recolección de información, explotando la capacidad que tienen los dispositivos móviles para obtener datos a través del uso de los sensores con los que cuentan [1]. También es posible recopilar metadatos sobre las mediciones que se realizan como son la ubicación, opiniones y experiencias de los usuarios [16]. La información recolectada mediante el MCS es la base para el desarrollo de servicios y aplicaciones en la novedosa área del Internet de las Cosas (en inglés, Internet of Things, IoT).

Los dispositivos móviles y su capacidad para obtener información del medio, así como su capacidad para compartirla, son elementos clave en el paradigma MCS. Además de los dispositivos móviles diseñados para uso industrial o especifico, son de especial interés aquellos que se utilizan a un nivel personal como son los teléfonos inteligentes, tabletas, relojes inteligentes, sistemas embebidos de videojuegos (Wii, Xbox Kinect) y altavoces inteligentes (Google Home, Amazon Echo), debido a las capacidades de procesamiento que poseen y a su bajo costo.

Los teléfonos inteligentes han mostrado una evolución constante desde el año 2010, integrando mejoras en su sistema operativo y aumentando los sensores que incluyen en su hardware. El equipamiento de sensores depende de la marca y modelo del teléfono inteligente, sin embargo, algunos de los sensores que podemos encontrar en los celulares de gama media de modelos lanzados en el año 2018 son: cámara, micrófono, barómetro, podómetro, GPS, acelerómetro, brújula, giroscopio, intensidad de luz ambiental y sensores biométricos (lector de huella digital). Adicionalmente, los teléfonos inteligentes cuentan con diversos mecanismos para poder compartir las mediciones realizadas además de la red celular, como son el WiFi, Bluetooht, NFC y cable USB. Las características que poseen los teléfonos inteligentes como su nivel de equipamiento de sensores, facilidades que poseen para comunicar datos y su uso generalizado entre las personas, los convierten en la elección frecuente para aplicaciones de MCS donde se requiere recopilación de datos masiva a un bajo costo [9].

Existen diversas áreas donde se han desarrollado aplicaciones que utilizan el paradigma de MCS utilizando solamente teléfonos inteligentes como medios para la recopilación de datos. En el ámbito ambiental, por ejemplo, se ha utilizado el MCS en el desarrollo de sistemas para la medición de contaminantes en el aire [8,12], de niveles de ruido [5] o condiciones climatológicas[13]. El paradigma MCS ha sido utilizado para desarrollar aplicaciones orientadas hacia ciudades inteligentes como lo es el monitoreo del tráfico para tener transportes eficientes [17] y monitoreo de vialidades con dañadas [10]. Otra área de aplicación es la salud, donde se han desarrollado sistemas para el monitoreo de la actividad física de las personas [2], de hábitos alimenticios [4] y monitoreo de datos relacionados con diversas enfermedades [6,11]. En áreas como el comercio [15] y turismo [18,14] también se han desarrollado aplicaciones que utilizan teléfonos inteligentes bajo un esquema de MCS.

Las aplicaciones desarrolladas bajo el paradigma de MCS se pueden catalogar dentro de dos categorías, en términos de la forma en que los participantes realizan 
la recopilación de los datos [7]: 1) monitoreo participativo (en inglés, Participatory Sensing) y 2) monitoreo oportunista (en inglés, Opportunistic Sensing).

El monitoreo participativo se caracteriza porque el participante actúa con iniciativa propia en la recopilación de los datos, asumiendo la responsabilidad de la información que se recopila y la forma que se lleva a cabo esta acción. El monitoreo participativo, requiere que el participante asuma una postura activa dentro del proceso de recopilación de los datos y no requiere de una infraestructura adicional para realizar este proceso $[3,7]$.

El monitoreo oportunista se caracteriza porque requiere de un conjunto de aplicaciones preinstaladas en el dispositivo móvil que realicen una serie de acciones, que se ejecutan en segundo plano, para la recopilación de datos. En el monitoreo oportunista los datos se recopilan de manera automática y no requiere de una intervención activa del participante [1].

El factor humano es un elemento importante en el paradigma MCS que requiere de la definición de criterios para su selección y regular su participación el monitoreo de datos. En el trabajo [1] se presentan los siguientes aspectos a considerar para una adecuada elección de participantes: a) se deben considerar un número suficiente de participantes por locación; b) se requiere definir incentivos que motiven la participación del factor humano en el proceso de recopilación de datos y garantice la calidad del proceso; c) se requiere de mecanismos de seguridad que protejan la ubicación de los participantes; d) se requiere que los participantes conozcan los mecanismos que proveen los teléfonos inteligentes para compartir datos (WiFi, Bluetooth, datos celulares, entre otros).

\section{Monitoreo biológico de cuerpos de agua}

Las fuentes de agua superficiales, representan el pilar de desarrollo de las diferentes actividades socioeconómicas, realizadas en las diferentes poblaciones alrededor del planeta, sin embargo, muchas de estas actividades provocan alteraciones e inclusive deterioro de la calidad de las fuentes de agua. En general, los cuerpos de agua superficiales están sometidos a dos tipos de contaminación: la primera, contaminación natural (provocada por el arrastre de materia particulada y disuelta en presencia de materia orgánica natural) y la segunda, de origen antrópico (descarga de aguas residuales domésticas, actividades agrícolas, actividades industriales, etc.). Diversos problemas se originan debido al mal estado de cuerpos de agua, por ejemplo, el mayor impacto sobre salud pública, tiene su origen a través de los sistemas de abastecimiento de agua; el cual es definido, como riesgo de transportar agentes contaminantes que puedan provocar enfermedades.

En la mayoría de países en vías de desarrollo (incluyendo México), el riesgo microbiológico es bastante elevado, aunado a una mala sanidad, dato que se ratificó en la Agenda 21 de la Conferencia de Naciones Unidas sobre Medio Ambiente y Desarrollo, en la cual afirma que aproximadamente $80 \%$ de todas las enfermedades, y más de la tercera parte de los decesos en estos países (en vías de desarrollo), se tienen a causa del consumo de agua contaminada. El notable 
deterioro de cuerpos de agua, provocó que se torne prioritario el monitoreo de la calidad de aguas superficiales, con el fin de tomar medidas de control y reducción del nivel de riesgo, factores que serán determinantes en la complejidad y costos de los tratamientos o medidas a tomar.

Para la determinación de la calidad de cuerpos de agua, se determinaron diversos factores, tales como los usos del suelo, la producción industrial y agrícola en áreas aledañas, e incluso, la misma cantidad de agua en ríos, lagos y lagunas. Con la finalidad de evaluar la calidad o también llamado grado de contaminación del agua, se han desarrollado índices de calidad, tanto generales, como específicos. En México se emplea un método llamado, Índice de Calidad del Agua (ICA), que agrupa algunos parámetros del deterioro de la calidad del líquido ${ }^{1}$. Para la evaluación, el índice toma valores en una escala de 0 a $100 \%$, en donde mientras mayor sea el valor, la calidad aumenta. El ICA se calcula a partir de la ponderación de 18 parámetros fisicoquímicos, entre los cuales resaltan la demanda bioquímica de oxígeno (DBO), oxígeno disuelto, coliformes, fosfatos, pH, sólidos suspendidos. Una de las desventajas del ICA es el costo que conlleva su implementación en los cuerpos de agua, que requiere de reactivos, instrumentos especializados y técnicos para su manipulación.

Una alternativa es el para el monitoreo biológico de los cuerpos de agua es el Biological Monitoring Working Party (BMWP por sus siglas en inglés). El índice BMWP utiliza principalmente la presencia de macroinvertebrados (organismos invertebrados de tamaño suficientemente grande para ser vistos sin el uso de microscopio) para determinar la calidad de un cuerpo de agua, debido a que los macroinvertebrados tienen diferentes grados de tolerancia a distinto tipos de contaminación. Los macroinvertebrados son generalmente sedentarios, tienen un ciclo de vida largo y son sensibles a las sustancias presentes en el agua. Las características de los macroinvertebrados permiten que el índice BMWP sea una alternativa confiable y económica para el monitoreo de cuerpos de agua al no requerir de equipo especial. El índice debe ser calibrado y validado para cada región ecológica en virtud de las importantes diferencias en la composición taxonómica, clima, y a los cambios en el nivel de sensibilidad de los organismos indicadores (macroinvertebrados acuáticos).

Por otro lado, a la fecha, en México, la normativa para la evaluación de la calidad el agua no cuenta con el uso de bioindicadores, los cuales son ampliamente usados en países desarrollados como el caso de Estados Unidos y la Unión Europea, así como otros países latinoamericanos, Costa Rica y Panamá. De igual forma, otros países han establecido el monitoreo participativo con apoyo de las poblaciones locales, las cuales realizan de forma voluntaria la vigilancia de sus recursos. Entre los programas internacionales se encuentran: El Programa de monitoreo voluntario Adopta tu cuenca de la Agencia de Protección Ambiental de Estados Unidos de América (EUA) ${ }^{2}$. El Programa voluntario de Alabama Water

\footnotetext{
${ }^{1}$ http://dgeiawf .semarnat.gob.mx:8080/ibi_apps/WFServlet?IBIF_ex=D3_R_ 
Watch $^{3}$ en USA, que forma parte de la amplia red de Global Water Watch de la Universidad de Auburn, Alabama ${ }^{4}$. Monitoreo comunitario participativo en Canadá que tiene apoyo gubernamental (http://www.enr.gov.nt.ca/programs/ water-resources/community-based-monitoring). Programa de monitoreo comunitario de la calidad del agua en Australia ${ }^{5}$. A su vez, en América Latina algunos países como Brasil, Colombia y Costa Rica, cuentan con redes de monitoreo comunitario. Estos ejemplos de monitoreo participativo ponen de manifiesto la importancia de llevar a cabo el monitoreo comunitario que coadyuvan a la comprensión de la condición ambiental de las corrientes hidrográficas, y además permiten que las comunidades se apropien de las técnicas de monitoreo y utilicen la información generada (a la par con el conocimiento local existente) para sus necesidades. En México, donde se presencian múltiples problemáticas vinculadas con el recurso hídrico asociado a los cambios en el uso del suelo, que implican sobre todo la contaminación y abatimiento de las fuentes de abastecimiento de agua potable, hace falta la implementación del monitoreo participativo con un protocolo que permita abordar de forma sistemática la evaluación de la calidad del agua. Las Áreas Naturales Protegidas (ANPs) constituyen un modelo de estudio adecuado para poner a prueba el uso de indicadores biológicos a través del índice BMWP y el uso de aplicaciones móviles para el monitoreo participativo, ya que, además de contar con altos niveles de diversidad que pueden aportan a una calibración del índice BMWP con gran diversidad de familias, también cuentan con Planes de Manejo y de Programas de Vigilancia a través de la participación de la Comisión Nacional de Áreas Naturales protegidas. Por lo que en estas ANPs es posible trabajar en coordinación con la dirección de las ANPs para llevar a cabo el monitoreo participativo y generación de ciencia ciudadana. En este proyecto se seleccionarán como zonas de estudio a las ANPs: reserva de la biosfera Tehuacán Cuicatlán, Reserva de la biosfera Sierra Gorda y ANP Camécuaro y el río Duero.

\section{Propuesta de aplicación para el monitoreo de cuerpos de agua}

En este módulo, se describen los elementos que conforman el prototipo para el monitoreo de los cuerpos de agua. La propuesta se enfoca en el monitoreo colaborativo, por lo que, se requiere de un mecanismo que permita a colaboradores participar en la recolección de datos sobre el fenómeno de interés relacionado con el estado de los cuerpos de agua. En el prototipo se asume que los colaboradores no poseen conocimiento especializado en las TIC, sin embargo, se consideran nativos digitales con conocimientos básicos sobre el uso de teléfonos inteligentes (uso de interfaz gráfica y uso de cámara) que coincide con un escenario real.

El prototipo utiliza la estrategia del índice BMWP para determinar la calidad de un cuerpo de agua. El índice BMWP considera aspectos como tipo de

\footnotetext{
3 http://www .alabamawaterwatch.org

${ }^{4}$ http://www.globalwaterwatch.org/

${ }^{5}$ http://www. seqcatchments.com.au/programs
} 
macroinvertebrados presentes, tipo de sedimentación, variables climatológicas, tipo de cauce, entre otros, para evaluar la calidad de un cuerpo de agua.

La infraestructura propuesta se muestra en la figura 1. Para la plataforma móvil se propone desarrollar dos aplicaciones orientadas a teléfonos inteligentes. La primera aplicación, implementará el protocolo para la bioevaluación de la calidad de agua, mediante una serie de vistas que incluyen preguntas acerca de las características del cuerpo de agua y la identificación de organismo que se encuentran en el cuerpo de agua. La segunda aplicación, integrará la información recolectada por los colaboradores mediante el uso de la primera aplicación y del protocolo de Bluetooth, para realizar la transferencia de datos hacia la segunda aplicación. Será la segunda aplicación la responsable de enviar la información recolectada hacia un servidor central, debido a la falta de conectividad en la zona donde se encuentran los cuerpos de agua. Las aplicaciones serán utilizadas por colaboradores que radiquen cerca de los cuerpos de agua que se quieren analizar.

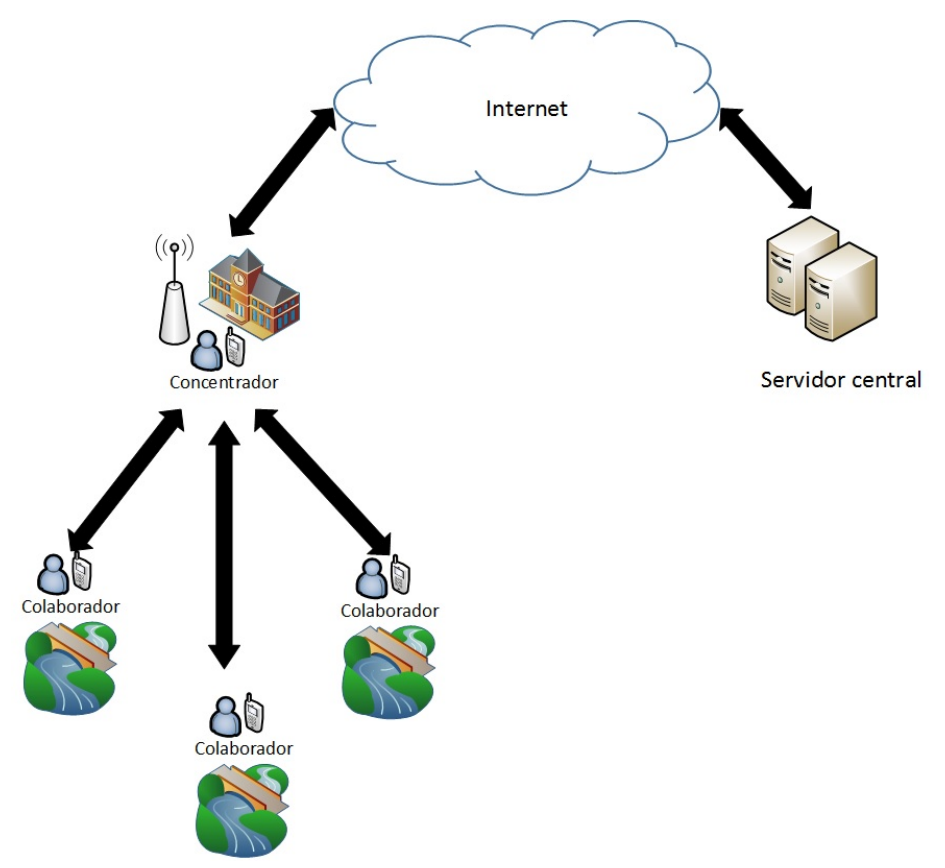

Fig. 1. Esquema general de la propuesta.

\section{Funcionamiento general de la aplicación móvil}

En la figura 2 se ejemplifica el funcionamiento general de la aplicación. Está dividida estratégicamente en etapas que nos permiten avanzar de manera sencilla 
y dinámica en la evaluación de los cuerpos de agua. En todas las etapas se proporcionará ayuda e información para facilitar la toma de decisiones, así como también evitar errores. En la primera etapa, de registro, al encontrarnos en un lugar sin acceso a internet se omitirá la fase de registro, rescatando únicamente los datos necesarios para la evaluación:

- Nombre de usuario,

- Nombre del río,

- Identificador,

- Municipio,

- Estado,

- Cuenca,

- Orden del río.

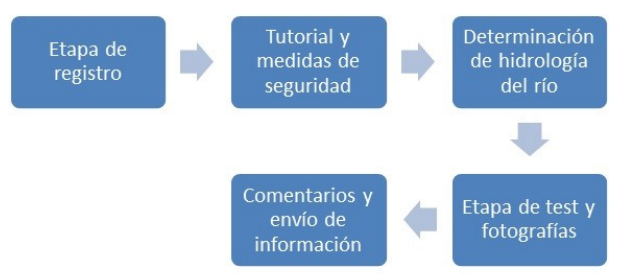

Fig. 2. Diagrama general del funcionamiento de la aplicación.

Los datos anteriores son los básicos necesarios para comenzar la evaluación. En la siguiente etapa, encontraremos las medidas de seguridad para realizar la evaluación, de igual manera, se presenta un tutorial, en el cual se describirá de manera sencilla el funcionamiento de la aplicación y la forma correcta de recopilar la información en los test que se encuentran en las siguientes etapas. La tercera etapa, presenta una pequeña encuesta, auxiliar en la determinación de la hidrología del río. Está información sirve como preámbulo y se complementará con la información recabada por los test. En la aplicación, inicialmente se desplegarán tres opciones con imágenes representativas (facilitadores para la toma de decisión), de las cuales, únicamente se podrá seleccionar una; dichas opciones abordan el tema de la fase acuática del río, las opciones son:

- El agua corre,

- El río presenta pozas,

- El río es seco.

Una vez seleccionada una opción, el siguiente paso, es determinar un régimen hidrológico de acuerdo a la época del año; para este proceso se desplegarán las 
cuatro estaciones del año consecutivas, de las cuales es necesario seleccionar al menos dos estaciones y como varió, si es que existió un cambio, la hidrología a través de las mismas, por ejemplo, en primavera el río se mantuvo con pozas, mientras que en verano el río se secó. Finalmente se procederá a determinar la tipología del río, de igual manera que en procesos anteriores, se desplegarán alrededor de 7 imágenes, cada una correspondiente a una tipología de un río.

Además de las imágenes, la aplicación presentará botones de información, con datos de relevancia, que sirvan como herramienta auxiliar para seleccionar la respuesta más adecuada, y por lo tanto, la evaluación sea precisa. La cuarta etapa, etapa de test, se podría contemplar como la más importante, ya que en esta se lleva a cabo el proceso de recopilación de información. Como primer paso, es necesario tomar una fotografía del río, de vital información para corroborar la información recaudada por los test. A continuación de la fotografía se presentan 3 test, los cuales son:

- Test de Estado Hidrológico,

- Test Hidroformológico,

- Test Biológico.

Cada test recauda información pertinente a su área, pero en conjunto nos ayudan a realizar un análisis de la calidad de cuerpos de agua, todo esto con la validación de un experto. En el test hidrológico, se busca obtener información acerca de:

- Infraestructura cercana,

- Extracción humana de agua,

- Cambios históricos del caudal del río.

Todo con la finalidad de determinar si estamos tratando un río que ha sido alterado por la actividad humana, o nos encontramos en un río que se ha mantenido intacto al contacto de las poblaciones. El segundo test, tiene como propósito conocer información acerca de:

- Naturaleza del río,

- Continuidad de la naturaleza,

- Paisaje,

- Canales fluviales,

- Contaminación.

$\mathrm{Y}$ finalmente, el tercer test, en el cual es necesario remover algunas rocas, recolectar alrededor de 50 macro invertebrados, los cuales, son determinantes para el análisis final. En este apartado, se desplegará una lista con las imágenes de cada invertebrado que se pudiera encontrar en el río, sin importar su calidad (buena, mala o regular). Es necesario seleccionar el invertebrado encontrado, así como tomar una fotografía del mismo, para proveer al experto, las herramientas necesarias para que el lleve a cabo se labor. 


\section{Características de la encuesta para la aplicación móvil}

La técnica de biomonitoreo de la calidad del agua, empleando macroinvertebrados acuáticos como bioindicadores, requiere de una recopilación primaria de características físicas y químicas del sitio de muestreo. Esta información le permitirá al grupo de expertos biólogos, interpretar de manera correcta y confiable, los resultados que emitirá la aplicación. Esta caracterización se basada en los Protocolos de Bioensayos Rápidos de la Agencia de Protección Ambiental de Estados Unidos (United States Enviroment Protection Agency, USEPA).

El proceso de caracterización comienza por delimitar un tramo del río a muestrear tomando una distancia de $100 \mathrm{~m}$, que cubra las características más representativas de cada hábitat y la variedad de condiciones físicas de la estación. Dicha caracterización consta de 4 pasos, los cuales se muestran al iniciar la aplicación móvil.

- Datos generales: Corresponde a la información general sobre el sitio de muestreo. Recopila información como: nombre del río, identificador ID del sitio, cuenca, orden del río, localidad más cercana, municipio, fecha del muestreo, hora del muestreo, altitud y coordenadas geográficas.

- Número de control: Corresponde a una numeración única que se asigna a la estación de muestreo.

- Condiciones climáticas: Se evalúa eventos como tormentas, nubosidad, lluvias intermitentes, temperatura ambiental y velocidad del viento (de poder contar con el dato), que pudieran afectar a la muestra.

- Características del cuerpo de agua: Dado que existe una estratificación natural de ensamble de macroinvertebrados, es importante tener en claro si la muestra es llevada a cabo en la parte alta (Crenon), media (Ritron) o baja de la cuenca (Potamon), esto a su vez corroborado por una fotografía tomada al momento y guardada en el formulario, que incluya las características del sitio en el tramo de 100 metros.

La segunda parte del formulario de la aplicación móvil, consiste en información más puntual acerca del estado del cuerpo de agua que está siendo muestreado. Apoyado a su vez por fotografías del sitio y de los macroinvertebrados encontrados. Los datos obtenidos en esta parte de la aplicación móvil tendrán una ponderación, la cual permitirá obtener un puntaje al final de la sesión, que servirá a los expertos como un referente, antes de que den el visto bueno y califiquen el estado de la calidad del agua en dicho sitio. La información (proporcionada por el área de biología) para tomar en cuenta en el formulario es la siguiente:

- Fauna: Sustrato/cobertura disponible. Se refiere a la diversidad de habitas dependientes del sustrato y la variedad de ellos presentes en el lecho del río. Los óptimos se caracterizan por ser altamente heterogéneos, mientras que los pobres contienen un solo tipo de hábitat como arenas.

- Óptimo: más del $70 \%$ del sustrato favorable para la colonización fauna acuática. 
- Subóptimo: 40-70\% de hábitat estable; adecuado para el desarrollo de potencial colonización.

- Marginal: $20-40 \%$ de hábitat estable; se encuentra reducido por perturbación o remoción.

- Pobre: menos de un $20 \%$ de hábitats estables, poca disponibilidad de colonización.

- Sustrato/tipo: En esta sección, se requiere definir la proporción de tamaño de partículas disponible en el sustrato y la variedad en la proporción alcanzará las máximas calificaciones, por el contrario, la proporción predominante de arena alcanzará calificaciones más bajas.

- Óptimo: 0-25\% de gravas, cantos y partículas está rodeada de sedimento fino.

- Subóptimo: 25-50 de la superficie de rocas, cantos y grava rodeadas de sedimento fino.

- Marginal: $50-75 \%$ de la superficie de rocas, cantos y grava rodeadas de sedimentos fino. Sin vegetación sumergida.

- Pobre: más del $75 \%$ de la superficie de rocas, cantos y grava rodeadas de sedimento fino. Sin vegetación o raíces.

- Relación profundidad y velocidad: Hace referencia a los cuatro patrones de velocidad y profundidad que se encuentran en corrientes de alto gradiente. Las pautas generales son: $0.5 \mathrm{~m}$ de profundidad para separar superficial de profundo y $0.3 \mathrm{~m} / \mathrm{s}$ para separar rápido de lento.

- Óptimo: Cuatro combinaciones: (1) lento-profundo, (2) lento-somero, (3) rápido-profundo y (4) rápido-somero.

- Subóptimo: Tres combinaciones. La ausencia de rápido/bajo determina menor puntaje

- Marginal: Dos combinaciones. La ausencia de rápido/bajo y lento/bajo determina menor puntaje.

- Pobre: Una sola combinación presente. Usualmente está presente lento/profundo.

- Depósito de sedimento: Evalúa la cantidad de sedimento que se ha acumulado, y los cambios que se han producido en el fondo de la corriente como resultado de la alteración de las riveras.

- Óptimo: Poco depósito sin islas ni barras, menos del $5 \%$ del fondo afectado por la deposición de sedimentos.

- Subóptimo: Formación de barras de grava, arena o sedimento, 5 a $30 \%$ del fondo afectado por la deposición de sedimentos; ligera deposición en los pozos.

- Marginal: Presencia de barras y 30 a $50 \%$ del fondo afectado. Sedimento sobre obstrucciones, constricciones y recodos. Moderada deposición en pozos.

- Pobre: Alto depósito de material y sin pozas. Mas del $50 \%$ del fondo cambia con frecuencia.

- Estado del canal: Evalúa el grado en que el canal está lleno de agua. El estado del flujo cambiará a medida que el canal se amplíe o cuando el flujo disminuya, como resultado de las presas y otras obstrucciones, desviaciones 
para el riego o sequía. Cuando el agua no cubre gran parte del lecho del río, la cantidad de sustrato adecuado para los organismos acuáticos es limitada.

- Óptimo: El nivel de agua alcanza la base de los bancos y el sustrato se encuentra infra expuesto.

- Subóptimo: El agua cubre más del $75 \%$ del cauce o menos del $25 \%$ del sustrato de fondo que expuesto.

- Marginal: El nivel del agua cubre entre el 25 al $75 \%$ del cauce y queda expuesta la mayor parte del sustrato de los rápidos.

- Pobre: Muy poca agua sobre el cauce, casi como pozas.

- Alteración del cauce: Es una medida de los cambios a gran escala en la forma de la corriente del canal. Muchos arroyos en áreas urbanas y agrícolas han sido enderezados, profundizados o desviados a canales concretos, a menudo para control de inundaciones o para fines de riego. Tales arroyos tienen mucho menos hábitats naturales disponibles para ser colonizados.

- Óptimo: Sin canalización o evidencia de ello, sustrato favorable para la colonización.

- Subóptimo: Alguna alteración o evidencia de ello, sin alguna canalización reciente.

- Marginal: Canalización extensiva con bancos o playas y de 40 a $80 \%$ de canalizado. Se puede considerar la presencia de barreras o diques.

- Pobre: Más del $80 \%$ del trecho del río canalizado y alterado. Márgenes protegidas con gaviones o cemento.

- Obtención de muestras de macroinvertebrados: En esta parte de la encuesta, se presentan imágenes alusivas a los macroinvertebrados más comunes que habitan en cuerpos de agua y cada uno cuenta a su vez con una ponderación definida, de acuerdo a su persistencia en los diferentes tipos de calidad de agua, con el propósito de que el usuario seleccione la opción que sea o se parezca al animal muestreado. Para efectos prácticos, se propone tomar una fotografía del animal para su verificación en el centro de control por un experto.

Como se comentó previamente, el ponderado final les dará a los expertos un parámetro con el cual dar un veredicto acerca de la calidad del cuerpo de agua.

\section{Uso de los datos recopilados}

Los datos obtenidos a partir de la encuesta, principalmente, serán usados para dar un panorama de la calidad del agua en una zona, pero, pueden ser usados para cubrir otras necesidades, o incluso, pueden proporcionar información adicional importante para los expertos.

Individualmente, cada encuesta servirá para determinar la calidad del agua en un determinado, río, lago, o algún otro tipo de cuerpo de agua, esto se hará, proporcionando un puntaje a los resultados obtenidos, pero este puntaje, tendrá como objetivo, asignar un color que indique que tan buena o mala es la calidad del cuerpo de agua, teniendo el rojo como la peor calidad y el azul como excelente calidad. 
La base de datos, recopilará información importante de cada río o lago de la zona, entre esta información, se encuentra la ubicación y fecha. Una vez que se haya recopilado la información de cada zona, se podrá procesar la información, y a partir de los datos obtenidos, se determinará en que zona se encuentra mayormente contaminada el agua, además, en qué fechas se cumple esto, ya que, las condiciones del medio ambiente, cambian a lo largo del año. Cabe destacar que, la encuesta y el puntaje asignado a cada organismo, puede cambiar a lo largo del año.

Adicionalmente, se podrá procesar la información acerca de los macroinvertebrados acuáticos presentes en el lugar, y analizar si alguno de ellos aparece demasiado seguido, o, por el contrario, si aparece muy pocas veces, o incluso nunca. Esta información es adicional, pero puede ayudar a los expertos, a conocer, o alertar si hay alguna anomalía en cuanto a la presencia de algún organismo en específico. Expresando lo anterior en otras palabras, se podrá tener estadísticas de la presencia de cada organismo, para poder determinar si su ciclo de vida se está efectuando de manera correcta.

Dejando de lado los datos, se tienen las fotografías, las cuáles son parte esencial de cada encuesta, ya que, si el usuario no está seguro de si un organismo es o no el mostrado en la aplicación, tendrá total libertad de tomar una foto al organismo, y esta podrá ser avalada por un experto. Teniendo clara la importancia de las fotografías, se llega a una parte importante en cuanto al procesamiento de la información, ya que, el usuario puede tomar una fotografía en la cual, en el fondo, haya objetos irrelevantes para la fotografía, o incluso, puede que el organismo se vea muy pequeño en comparación al tamaño de la toma, es por esto que, se realizará un proceso que pueda recortar las fotografías, de forma que, se reduzca en medida de lo posible, la información irrelevante que haya en ésta, y así, lo que prevalezca sea la imagen del organismo.

Resumiendo, los datos obtenidos, serán procesados para cubrir las necesidades primarias del proyecto, pero, proporcionarán información adicional a los expertos, que puede resultar muy útil para ellos. En cuanto las imágenes, serán procesadas para que, en cada fotografía, se aprecie mayormente el organismo, y no otra información irrelevante, además de que, también serán procesadas para reducir la cantidad de memoria requerida para ser almacenadas.

\section{Conclusiones y trabajo futuro}

La propuesta busca desarrollar una aplicación que permite realizar el monitoreo de cuerpos de agua mediante el uso del paradigma de monitoreo colaborativo móvil (MCS). El enfoque de MCS ofrece una alternativa viable para el monitoreo de las características de los cuerpos de agua de una región a través de la participación ciudadana, presentando las siguientes ventajas: 1) permite implementar el índice BMWP para determinar la calidad de los cuerpos de agua en términos de bioindicadores, 2) la propuesta promueve la participación ciudadana haciendo que éstas sean responsables de sus propios recursos, 3) permite integrar la información sobre los diversos cuerpos de agua en una sola red a nivel nacional 
y 4) permite identificar los sitios que requieren de atención para implementar estrategias de conservación, restauración o recuperación.

Como trabajo futuro se propone desarrollar módulos que permitan implementar técnicas de inteligencia artificial para obtener tendencias sobre el estado de los cuerpos de agua y técnicas de para el reconocimiento semiautomático de los macroinvertebrados recolectados.

Agradecimientos. Este trabajo ha sido realizado gracias al apoyo del Instituto Politécnico Nacional (Proyecto Multired 2045 Módulo IV con registro 20196153 de la SIIP-IPN).

\section{Referencias}

1. Abualsaud, K., Elfouly, T.M., Khattab, T., Yaacoub, E., Ismail, L.S., Ahmed, M.H., Guizani, M.: A survey on mobile crowd-sensing and its applications in the iot era. IEEE Access 7, 3855-3881 (2019)

2. Denning, T., Andrew, A., Chaudhri, R., Hartung, C., Lester, J., Borriello, G., Duncan, G.: Balance: towards a usable pervasive wellness application with accurate activity inference. In: Proceedings of the 10th workshop on Mobile Computing Systems and Applications. p. 5. ACM (2009)

3. Ganti, R.K., Ye, F., Lei, H.: Mobile crowdsensing: current state and future challenges. IEEE Communications Magazine 49(11), 32-39 (2011)

4. Gao, C., Kong, F., Tan, J.: Healthaware: Tackling obesity with health aware smart phone systems. In: 2009 IEEE International Conference on Robotics and Biomimetics (ROBIO). pp. 1549-1554. Ieee (2009)

5. Hachem, S., Mallet, V., Ventura, R., Pathak, A., Issarny, V., Raverdy, P.G., Bhatia, R.: Monitoring noise pollution using the urban civics middleware. In: 2015 IEEE First International Conference on Big Data Computing Service and Applications. pp. 52-61. IEEE (2015)

6. Jin, Z., Oresko, J., Huang, S., Cheng, A.C.: Hearttogo: a personalized medicine technology for cardiovascular disease prevention and detection. In: 2009 IEEE/NIH Life Science Systems and Applications Workshop. pp. 80-83. IEEE (2009)

7. Lane, N.D., Miluzzo, E., Lu, H., Peebles, D., Choudhury, T., Campbell, A.T.: A survey of mobile phone sensing. IEEE Communications magazine 48(9), 140-150 (2010)

8. Leonardi, C., Cappellotto, A., Caraviello, M., Lepri, B., Antonelli, F.: Secondnose: an air quality mobile crowdsensing system. In: Proceedings of the 8th Nordic Conference on Human-Computer Interaction: Fun, Fast, Foundational. pp. 1051-1054. ACM (2014)

9. Liu, J., Shen, H., Narman, H.S., Chung, W., Lin, Z.: A survey of mobile crowdsensing techniques: A critical component for the internet of things. ACM Transactions on Cyber-Physical Systems 2(3), 18 (2018)

10. Mohan, P., Padmanabhan, V.N., Ramjee, R.: Nericell: rich monitoring of road and traffic conditions using mobile smartphones. In: Proceedings of the 6th ACM conference on Embedded network sensor systems. pp. 323-336. ACM (2008)

11. Oliver, N., Flores-Mangas, F.: Healthgear: Automatic sleep apnea detection and monitoring with a mobile phone. JCM 2(2), 1-9 (2007) 
12. Pan, Z., Yu, H., Miao, C., Leung, C.: Crowdsensing air quality with camera-enabled mobile devices. In: Twenty-Ninth IAAI Conference (2017)

13. Pankratius, V., Lind, F., Coster, A., Erickson, P., Semeter, J.: Mobile crowd sensing in space weather monitoring: the mahali project. IEEE Communications Magazine 52(8), 22-28 (2014)

14. Pereira, F.C., Vaccari, A., Giardin, F., Chiu, C., Ratti, C.: 19 crowdsensing in the web: Analyzing the citizen experience in the urban space. From Social Butterfly to Engaged Citizen: Urban Informatics, Social Media, Ubiquitous Computing, and Mobile Technology to Support Citizen Engagement p. 353 (2011)

15. Sehgal, S., Kanhere, S.S., Chou, C.T.: Mobishop: Using mobile phones for sharing consumer pricing information. In: Demo Session of the Intl. Conference on Distributed Computing in Sensor Systems. vol. 13 (2008)

16. Sherchan, W., Jayaraman, P.P., Krishnaswamy, S., Zaslavsky, A., Loke, S., Sinha, A.: Using on-the-move mining for mobile crowdsensing. In: 2012 IEEE 13th International Conference on Mobile Data Management. pp. 115-124. IEEE (2012)

17. White, J., Thompson, C., Turner, H., Dougherty, B., Schmidt, D.C.: Wreckwatch: Automatic traffic accident detection and notification with smartphones. Mobile Networks and Applications 16(3), 285-303 (2011)

18. Wise, N., Heidari, H.: Developing smart tourism destinations with the internet of things. In: Big Data and Innovation in Tourism, Travel, and Hospitality, pp. 21-29. Springer (2019) 